\author{
Giovanna Colombetti \\ University of Exeter (UK)
}

\title{
Reply to Barrett, Gendron \& Huang
}

Barrett, Gendron \& Huang and I agree on more points than may appear at first sight. I fully endorse their claim that emotions are complex context-dependent and highly variable phenomena, and that the "affect program" framework (or any other account that posits emotions as internal causes of behaviour, expression, etc.), including the view that there is a limited number of "basic" affect programs, has difficulties accounting for this complexity. We also agree that Darwin and James were not "basic emotion" theorists, and that talk of "brain loci" for alleged basic emotions is inappropriate. These agreements aside, I shall point out that I never intended to provide a natural kind account of emotions, and that there are still various aspects of the Conceptual Act Model I find unclear. But I am ultimately happy that Barrett, Gendron \& Huang agree with me that a dynamical system framework is a promising avenue for further research on emotion.

My references to Darwin and James in the original paper are not meant to support the view that there are basic emotions. My point is rather that Darwin's legacy should not be identified with a Darwin-Tomkins-Ekman/Izard lineage (see section 6). Darwin did not talk of basic emotions, let alone affect programs (and I am happy to learn more about the origins of these notions). Darwin's legacy in emotion theory is, I want to suggest, best seen as the acknowledgement of the existence of coherent dynamical patterns in behaviour, physiology, expression, and even experience - a legacy continued and refined by James and Dewey 
(among others), and alive in current dynamical system approaches in affective science. As for James, my reference to his work is meant to support the claim that prior to the "affect program" framework, there were emotion theorists who saw emotions as associated with different neural and physiological processes, different functions and different experiences, and yet who did not talk of "internal causes". It is true that James wrote e.g. that "all sorts of groupings" of the emotions are possible, depending on one's concerns, projects, and available introspective vocabulary (James, 1890, p. 485); this and other similar passages (e.g. op. cit. p. 454) manifest James's pluralism, and his pragmatism about taxonomies (see also Charland, 2002; Hatfield, 2007; Ratcliffe, 2008). As I see it, my discussion is entirely consistent with both aspects of James's thought, as well as with his numerous descriptions of how e.g. "objects of rage, love, fear, etc., not only prompt a man to outward deeds, but provoke characteristic alterations in his attitude and visage, and affect his breathing, circulation, and other organic functions in specific ways" (James, 1890, p. 442).

Related to this point, the reason why my paper does not say anything about why English speakers tag a variety of behaviours, expressions, etc. as manifestations of e.g. "fear" is that I did not mean to address this issue in the first place. I am not seeking to identify essences, entities, or even patterns that would correspond to English emotion terms. Rather, my aim in the paper is to illustrate the usefulness of notions such as self-organization and meta-stability when it comes to describing emotional episodes, against the claim that there are no coherent and recurrent organismic patterns that can be said to correspond to emotional episodes. The relevant fact is that English speakers, as well as non-English speakers and nonspeakers, manifest, experience, recognize and respond to reliably recurrent patterns, which I have called "emotion-forms".

I do however agree with Barrett and colleagues that words can do many interesting things to our organism and feelings (including constituting, clarifying and enhancing 
experience, as well as providing ecological niches for the development of culture-specific emotions; see Colombetti, forthcoming), and that is precisely thanks to the openness and pliability of dynamical emotion-forms. As for the role of categorization in emotions, I think there are still several aspects of their proposal that Barrett and her colleagues need to clarify. For one, they seem to be conflating two levels of description that ought to be kept distinct. On the one hand, they call "categorization" the brain's continuous processing of information about the body as well as objects external to it, and the neural re-structuring, pruning, etc. that take place during this processing. This is a subpersonal form of "categorization", which I would rather describe as the continuous realization of various organismic patterns, i.e. the enactment of organismic forms in virtue of the coupling of brain, body and world. At this subpersonal level of description, our differences are perhaps merely terminological. On the other hand, Barrett, Gendron \& Huang also talk of categorization as "what people know about emotion", as a process that makes use of "conceptual knowledge for emotion". If by this they mean that personal-level conceptual knowledge is necessary to experience specific emotions, and to respond to emotions in other agents, then I think their claim is too radical. I would rather want to argue that organismic patterns are directly felt as meaningful by the organism undergoing them, i.e. without the interposition of conceptual knowledge and/or "mental tools" (for some related arguments, see Colombetti, 2007); and that responding to others' emotions can also happen without conceptual knowledge. None of this would of course imply that people's knowledge, narratives and values do not affect how they experience emotions. But this is just a vague answer, and a more precise discussion could only follow from a clearer account of what Barrett, Gendron \& Huang mean by "conceptual knowledge" and its relation to categorization.

I did not intend to propose "yet another natural kind account" of emotions. I never use the term "natural kinds" in the paper, because there is no agreement in the philosophical 
literature on what counts as a natural kind, and my paper is not aimed at engaging with this (complex) debate. All I will say here, then, is that Barrett, Gendron \& Huang seem to be confusing the question of whether emotion words pick out some sort of "deep essence" with the question of whether emotions are natural kinds. One can hold the latter view and reject the former, and indeed some philosophers hold non-essentialist views of natural kinds (e.g. Dupré, 1993). On one characterization (one that has been mentioned various times in empirically minded philosophical discussions of emotions, see Charland, 2002; Griffiths, 1997; Prinz, 2004), natural kinds are homeostatic property clusters that can vary with context, and that at the same time maintain a stable range of properties (Boyd, 1991). It strikes me as an interesting question whether or not what I have called "dynamical discrete emotions" satisfy Boyd's account of natural kinds, and more in general whether or not a dynamical system account of emotions is indeed "yet another natural kind account" of emotions.

Finally, I am happy that Barrett, Gendron \& Huang agree with me that what I have called "dynamical affective science" is a promising avenue for further research on emotions-even though they seem to think I am proposing a "model" (whereas I am just drawing attention to a relatively neglected approach in affective science, and explaining why I think it provides a viable and useful alternative to existing approaches), and even though they do not comment on the current "dynamical" empirical research on emotion I discuss in the second part of the paper. This research (and in fact all the dynamical system approaches to emotion I am familiar with) does point to the existence of variable context-dependent organismic patterns, and patterned variability is a recurrent theme in dynamical affective science (see references in original paper). Empirical work in dynamical affective science supports my suggestion that emotional episodes are best seen as meta-stable configurations of various processes spanning brain and body. This suggestion by no means "locates" emotions in alleged dedicated brain centres; rather, it characterizes emotional episodes as trajectories in 
a very complex state space representing the whole organism. Although I do not discuss these further issues in the paper, the point of this characterization is to allow emotional episodes to be perturbed and modified in concrete intersubjective encounters, and in virtue of their being situated in a shared context of norms and symbols.

References (excluding those already listed in original paper)

Boyd, R. (1991). Realism, anti-foundationalism and the enthusiasm for natural kinds. Philosophical Studies, 61, 127-48.

Charland, L.C. (2002). The natural kind status of emotion. British Journal for the Philosophy of Science, 53, 511-37.

Colombetti, G. (forthcoming). What language does to feelings. Journal of Consciousness Studies.

Colombetti, G. (2007). Enactive appraisal. Phenomenology and the Cognitive Sciences, 6, $527-46$.

Dupré, J. (1993). The disorder of things: Metaphysical foundations of the disunity of science. Cambridge: Harvard University Press.

Hatfield, G. (2007). Did Descartes have a Jamesian theory of the emotions? Philosophical Psychology, 20, 413-40.

James, W. (1890). Principles of psychology (Volume II). New York: Holt.

Ratcliffe, M. (2008). Feelings of being: Phenomenology, psychiatry and the sense of reality. Oxford: Oxford University Press. 